\title{
A Mathematical Model for Electrolocation in Weakly Electric Fishes
}

\author{
Thomas Boulier ${ }^{a}$ \\ Sorbonne Universités, UPMC Univ Paris 06, CNRS, INSERM, Laboratoire d'Imagerie Biomédicale (LIB), F-75013, \\ Paris, France
}

\begin{abstract}
Weakly electric fishes usually live in the rivers of Africa and South America. They are able to orient themselves thanks to an electric sense, called electrolocation, that consist of emitting an electric field and sensing it at the surface of their skin. Whenever an object is nearby the fish, it distorts the emitted electric field; this perturbation is recorded by the electroreceptors, hence allowing the fish to recognize the object.

Discovered in 1958 by the work of Hans Lissmann and Ken Machin, this electric sense has then been intensively studied. We now know that weakly electric fishes are able to locate obstacles with an accuracy of about one centimetre, and to differentiate between different shapes and/or materials. However, a fundamental question has risen from a physical point of view: how is it possible to recover such details with such a low-amplitude $(1 \mathrm{mV})$ and low-frequency $(1 \mathrm{kHz})$ signal?

Mathematical modelling of the electric field emitted by these fishes allow us to quantify, theoretically and numerically, the information contained into this signal. In this work, we propose to derive the equations governing the emitted-and-perturbed electric field of a fish; we also describe possible algorithms to recover the position and the shape of an object. In particular, we show that the use of multiple frequencies can give us the location of an object, and the use of movement can lead us to the differentiation between different shapes, into a machine-learning framework.
\end{abstract}

\section{References}

1. H. Ammari, T. Boulier, J. Garnier, SIAM J. Imaging Sci. 6 (2013)

2. H. Ammari, T. Boulier, J. Garnier, W. Jing, H. Kang, H. Wang, Found. Comput. Math. 14, 27 (2014)

3. H. Ammari, T. Boulier, J. Garnier, H. Wang, Proc. Natl. Acad. Sci. USA 111, 11652 (2014)

\footnotetext{
${ }^{\mathrm{a}}$ Corresponding author: thomas.boulier@polytechnique.edu
} 